\title{
SÍNTESIS Y CARACTERIZACIÓN DE BIOVIDRIOS DE FOSFATO POR EL MÉTODO PIRÓLISIS DE AEROSOL EN LLAMA
}

\author{
Juan Camilo Vélez ${ }^{1}$, Natalia Betancur ${ }^{2}$, Omar Gutiérrez ${ }^{1}$ \\ ${ }^{1}$ Grupo Alquimia, Facultad Ciencias Exactas y Aplicadas, Instituto Tecnológico Metropolitano, Medellín, Colombia. \\ 2 Departamento de materiales y minerales. Grupo de investigación de cemento y materiales de construcción \\ (CEMTCO), Universidad Nacional de Colombia, Calle 65 No 59 A-110, Medellín 050036, Colombia.
}

\section{RESUMEN}

Empleando el novedoso método pirólisis de aerosol en llama (FSP) se sintetizó por primera vez un biovidrio de fosfato de calcio en el sistema $\mathrm{P}_{2} \mathrm{O}_{5}-\mathrm{CaO}-\mathrm{Na}_{2} \mathrm{O}$. Las muestras obtenidas se caracterizaron mediante difracción de rayos $X(D R X)$ y espectroscopía infrarroja de reflectancia difusa (DRIFTS), sirviendo esta última para seguir la cinética de biomineralización in-vitro durante 15 días en fluido corporal simulado (SBF). Mediante DRX se validó la naturaleza amorfa del biovidrio exhibiendo la fase whitlockita. El análisis DRIFTS reveló las bandas características de los grupos fosfatos en un rango de alrededor de los 500 a $1400 \mathrm{~cm}^{-1}$. Respectivamente, la evolución de las bandas de fosfatos en las muestras sumergidas en SBF indican la formación de apatita, proceso que siguió una cinética de pseudo- primer-orden de Lagergren.

Palabras clave: biomaterial; biovidrio, pirólisis de aerosol en llama; apatita; cinética de biomineralización.

Recibido: 3 de diciembre de 2020. Aceptado: 15 de mayo de 2021

Received: December 3, $2020 . \quad$ Accepted: May 15, 2021

DOI: http://dx.doi.org/10.33571/rpolitec.v17n33a11

\section{SYNTHESIS AND CHARACTERIZATION OF PHOSPHATE BIOGLASSES BY THE FLAME AEROSOL PYROLYSIS METHOD}

\begin{abstract}
Using the novel flame aerosol pyrolysis method (FSP), a calcium phosphate bioglass was synthesized for the first time in the $\mathrm{P}_{2} \mathrm{O}_{5}-\mathrm{CaO}-\mathrm{Na}_{2} \mathrm{O}$. The samples obtained were characterized by $X$-ray diffraction(XRD) and diffuse reflectance infrared spectroscopy (DRIFTS), the latter serving to follow in-vitro biomineralization kinetics for 15 days in simulated body fluid (SBF). Using XRD, the amorphous nature of the bioglass was validated by exhibiting the whitlockite phase. The DRIFTS analysis revealed the characteristic bands of the phosphate groups in a range of about 500 to $1400 \mathrm{~cm}^{-1}$. Respectively, the evolution of the phosphate bands in the samples submerged in SBF indicate the formation of apatite, a process that followed a pseudo-first-order Lagergren kinetics.
\end{abstract}

Keywords: biomaterial; bioglass; aerosol pyrolysis in flame; apatite; biomineralization kinetics.

Cómo citar este artículo: J. C. Camilo-Vélez, N. B. Betancur, O. Gutiérrez (2021). "Síntesis y caracterización de biovidrios de fosfato por el método pirólisis de aerosol en llama," Revista Politécnica, vol. 17, no. 33, pp. 126132, 2021. DOI: http://dx.doi.org/10.33571/rpolitec.v17n33a11 


\section{INTRODUCCIÓN}

Actualmente, la necesidad de implantes biomédicos para reparar tejidos enfermos o perdidos presenta varias desventajas, como baja disponibilidad, problemas de osteointegración, altos costos en el sistema de salud de estos remplazos para satisfacer las demandas, entre otros [1],[2]. Buscando nuevas alternativas en materiales para implantes, los biovidrios se han consolidado como un gran candidato para el desarrollo de estos sustitutos gracias a sus propiedades de biocompatibilidad, bioactividad, osteoconductividad y buena unión directa con el hueso [3]. De forma general, los biovidrios se dividen en vidrios a base de sílice $\left(\mathrm{SiO}_{2}\right)$ y fosfato $\left(\mathrm{P}_{2} \mathrm{O}_{5}\right)$ [3]. Los biovidrios de sílice han demostrado ser altamente bioactivos y los vidrios de fosfato pueden ajustarse a la composición de la fase mineral del hueso, presentando además un amplio rango de velocidades de degradación, que puede ser modificado dependiendo de la composición química del sistema vítreo [4],[5],[6].

Habitualmente, los biovidrios pueden ser sintetizados por varios métodos tradicionales tales como fusión, solgel, hidrotermal, coprecipitación, entre otros; sin embargo, pese a ser métodos muy favorables en cuanto a resultados de pureza y homogeneidad, presentan muchos obstáculos debido a que son procesos lentos y que difícilmente permiten obtener estos sistemas a gran escala [7],[8],[9]. Desde un punto de vista práctico, estos procedimientos no suplen las demandas, por lo que surge el interés de explorar nuevas técnicas de síntesis de biovidrios que ayuden a mejorar su proceso de producción. Con base en esto, el objetivo de este estudio consistió en preparar biovidrios de fosfatos en el sistema $\mathrm{P}_{2} \mathrm{O}_{5}-\mathrm{CaO}-\mathrm{Na}_{2} \mathrm{O}$ por un método novedoso llamado pirólisis de aerosol en llama (FSP) que, gracias a su estructura y funcionamiento, ha mostrado la posibilidad de sintetizar micro y nanopartículas con tiempos de síntesis de un día en comparación a los 6-10 días típicos en las rutas químicas mencionadas anteriormente, disminuyendo costos de producción e impactos ambientales, además, contando con la cualidad de ser fácilmente escalable a nivel industrial. Así mismo, el biovidrio sintetizado se ha caracterizado estructural y espectroscópicamente, evaluando su bioactividad mediante pruebas de biomineralización en fluido corporal simulado.

\section{MATERIALES Y MÉTODO}

\subsection{Materiales}

Fosfato de amonio $\left(\mathrm{NH}_{4}\right)_{3} \mathrm{PO}_{4}$ (Panreac, $\left.96 \%\right)$, nitrato de sodio $\mathrm{NaNO}_{3}(98 \%$, Sigma-Aldrich), nitrato de calcio tetrahidratado $\mathrm{Ca}\left(\mathrm{NO}_{3}\right)_{2} .4 \mathrm{H}_{2} \mathrm{O}$ (Sigma-Aldrich, $98 \%$ ), etanol absoluto (Merck), cloruro de sodio $\mathrm{NaCl}(98 \%$, Sigma-Aldrich), ), cloruro de potasio $\mathrm{KCl}\left(98 \%\right.$, Sigma-Aldrich), fosfato dipotásico trihidratado $\mathrm{K}_{2} \mathrm{HPO}_{4} \cdot 3 \mathrm{H}_{2} \mathrm{O}$ (98\%, Sigma-Aldrich), cloruro de magnesio hexahidratado $\mathrm{MgCl}_{2} \cdot 6 \mathrm{H}_{2} \mathrm{O}(98 \%$, Sigma-Aldrich), ácido clorhídrico $\mathrm{HCl}\left(98 \%\right.$, Sigma-Aldrich), cloruro de calcio $\mathrm{CaCl}_{2}\left(98 \%\right.$, Sigma-Aldrich), Sulfato de sodio $\mathrm{Na}_{2} \mathrm{SO}_{4}(98 \%$, Sigma-Aldrich), tris(hidroximetil) aminometano (98\%, Sigma-Aldrich) y agua desionizada (ProtoKimica).

\subsection{Síntesis mediante Pirólisis de aerosol en llama (FSP)}

Empleando las cantidades estequiométricas adecuadas de fosfato de amonio, nitrato de calcio tetrahidratado y nitrato de sodio para obtener 10 gramos de biovidrio por el método FSP [10],[11] el montaje mostrado en la Figura 1, consistió en la formación de un aerosol con aire como gas de dispersión a una presión de $200 \mathrm{kPa}$ utilizando una pistola de aspersión con aire comprimido, el cual se podía variar entre 10 psi y 50 psi. Para esto se utilizó un compresor Michelin MCX 50. El sistema de alimentación de los precursores fue de $30 \mathrm{ml} / \mathrm{min}$ y es independiente de los conductos del gas de arrastre, los cuales se mezclan en el área de atomización para formar el aerosol. El aerosol se dirigió en forma transversal hacia una llama oxidante con un flujo de oxígeno de $0.019 \mathrm{~m}^{3} / \mathrm{min}$ y de acetileno de $0.011 \mathrm{~m}^{3} / \mathrm{min}$, con una proporción de $1: 1.67\left(\mathrm{C}_{2} \mathrm{H}_{2}: \mathrm{O}_{2}\right)$. La zona de reacción se formó con dos llamas cruzadas de un ángulo de $27^{\circ}$ utilizando dos mangos de soplete marca Victor ${ }^{\circledR}$ equipadas con lanzas de soldar número 1. 
Adicionalmente, el montaje contó con un sistema de enfriamiento por convección forzada y los productos de la reacción fueron colectados manualmente.

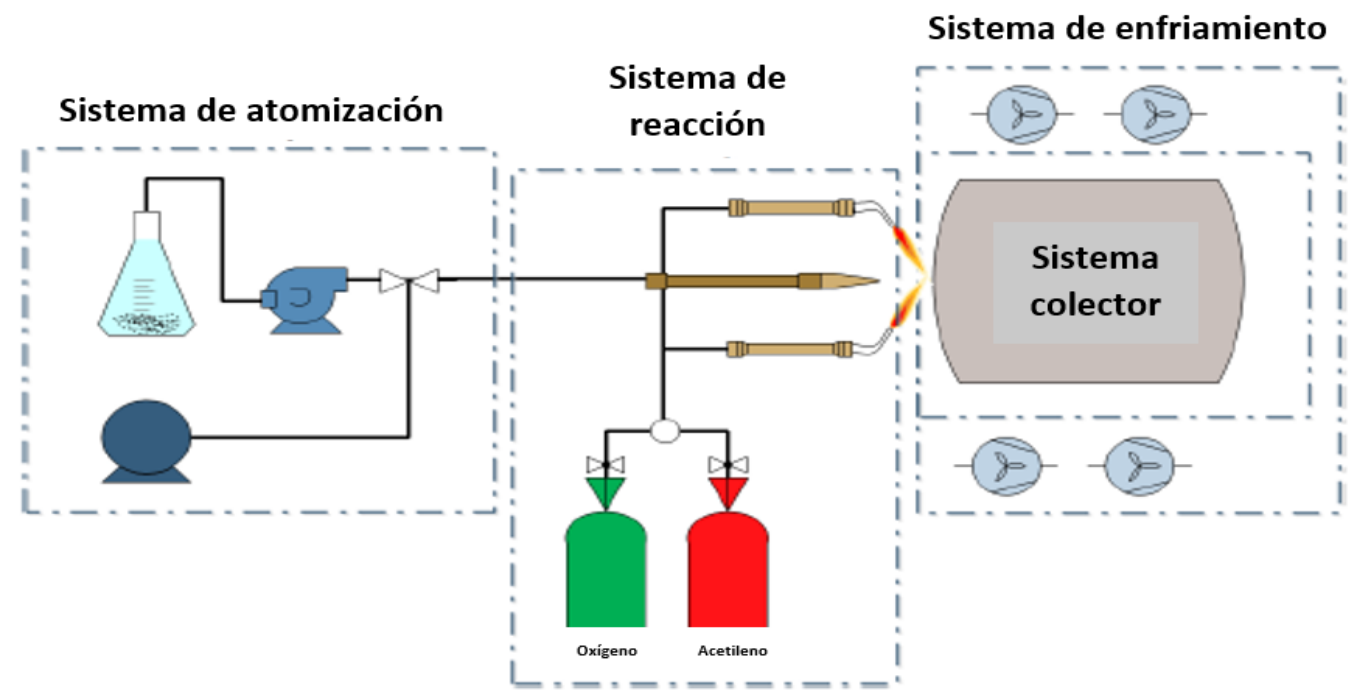

Figura 1. Esquema del método pirólisis de aerosol en llama (FSP). Tomado de [11].

\subsection{Caracterización}

Los polvos de biovidrio se caracterizaron mediante difracción de rayos $X$ (DRX) en un difractómetro Empyrean, Panalytical empleando un tamaño de paso de $0.05^{\circ}$ y 50 segundos por paso, además de un intervalo de ángulo $2 \theta: 5-60^{\circ}$. Las pruebas de biomineralización se realizaron mediante la inmersión del biovidrio en fluido corporal simulado (SBF) preparado con el protocolo de Kokubo [12]. Estas pruebas se realizaron en continuo con tiempos de muestreo progresivo durante 15 días. Los biovidrios expuestos al SBF fueron analizados mediante Espectroscopía Infrarroja en el modo de reflectancia difusa (DRIFTS) con un equipo Shimadzu - IRTracer-100, para seguir la evolución de las áreas de las bandas de los grupos fosfatos para describir de una forma cuantitativa la cinética del proceso de biomineralización. Para estos análisis se empleó una resolución de $4 \mathrm{~cm}^{-1}$, un intervalo de número de onda de 400-4000 $\mathrm{cm}^{-1}$ detectando el porcentaje de absorbancia, con 32 escaneos por muestra. La preparación de las muestras para el análisis en el equipo fue del $5 \% \mathrm{p} / \mathrm{p}$ respecto a la cantidad de $\mathrm{KBr}$.

\section{RESULTADOS Y DISCUSIÓN}

El método FSP implementado tuvo un rendimiento del 35\% con partículas de biovidrio con tamaños inferiores a $75 \mu \mathrm{m}$.

\subsection{Análisis difracción de rayos $X$}

La Figura 2 muestra el difractograma del biovidrio obtenido, en el que se puede verificar la naturaleza predominantemente amorfa del biovidrio. Así mismo, alrededor del intervalo $17^{\circ}$ a $35^{\circ} 2 \theta$, se observa un pico amplio que de acuerdo con la literatura está asociado a la fase whitlockita [13]. 


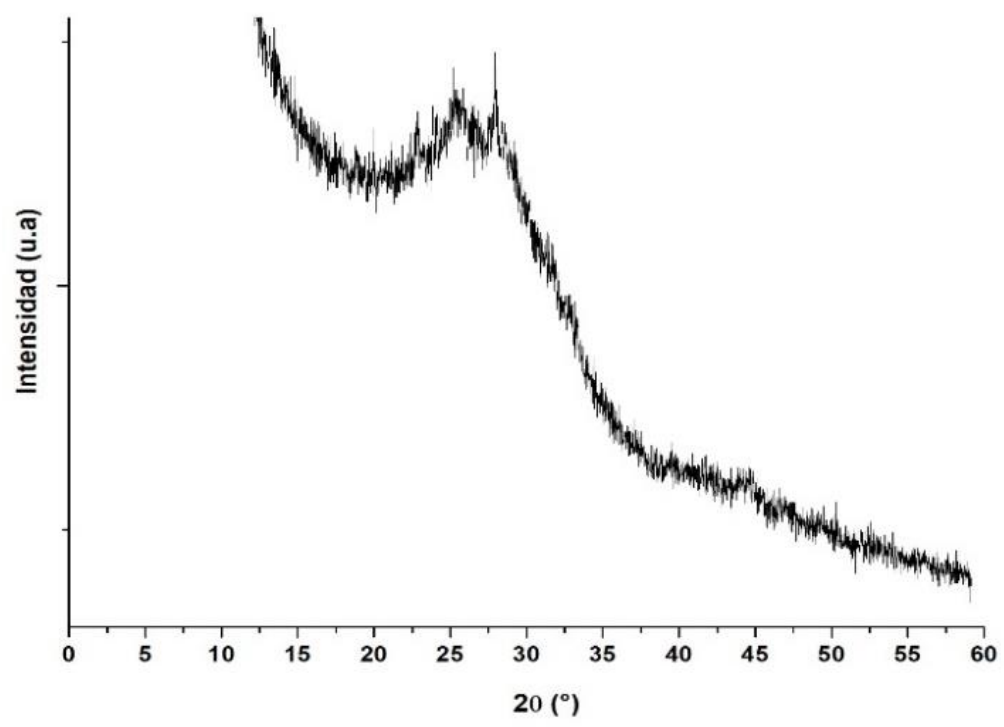

Figura 2. Espectro DRX del biovidrio derivado del método FSP.

\subsection{Análisis de ensayos de biomineralización mediante espectroscopía infrarroja}

Los espectros FTIR de las cinco muestras obtenidas, donde cada una de ellas manejaban una proporción de $0.05 \mathrm{~g}$ de biovidrio a razón de $20 \mathrm{ml}$ de SBF en un lapso de alrededor de 15 días y conservando una temperatura de $36.5^{\circ} \mathrm{C}$ a $37^{\circ} \mathrm{C}$ por medio de un horno calefactor se observan en la Figura 3a. En dicha figura, las bandas de interés corresponden a los grupos fosfatos en un rango a partir de los 500 a $1400 \mathrm{~cm}^{-1}$ [14], [15], donde visualmente se aprecian bandas bien definidas en cada uno de los espectros de las muestras analizadas en los diferentes días. La mayoría de las bandas características de este vidrio de fosfato de calcio permanecieron presentes a través del tiempo de inmersión en SBF. Adicionalmente, se observa que las intensidades relativas de cada una de las bandas dentro de cada espectro sufrieron cambios durante dicho periodo de inmersión.

Por otro lado, se observa en dicha figura, que las tres primeras bandas de fosfatos comprendidas en un rango de alrededor de 515 a $970 \mathrm{~cm}^{-1}$ y relacionadas a enlaces $\mathrm{P}-\mathrm{O}$, muestran un pequeño aumento en intensidad al transcurrir los días de inmersión en SBF, a excepción de la primera banda, donde en el día 4 disminuye la intensidad a tal forma de ser casi nula y en el día 14 la intensidad de esta es muy débil; además, un caso muy particular es la tercera banda, donde en el día 4 su intensidad es un poco más pronunciada que la del día 11, sin embargo, tanto en el día 11 como 14 esta banda toma una forma más definida. La cuarta banda de fosfato comprendida en un rango de alrededor de 1020 a $1265 \mathrm{~cm}^{-1} \mathrm{y}$ relacionada al grupo $\mathrm{PO}_{2}$, aparece de una forma más amplia en el día 2 comparada con las demás bandas en ese rango de los demás días, sin embargo, con los días transcurridos esta banda comienza a estrecharse y su intensidad aumenta notoriamente. Finalmente, a partir del día 11 se percibe la aparición de una banda débil en un rango de 1270 a 1390 $\mathrm{cm}^{-1}$ relacionada al grupo $\mathrm{P}=\mathrm{O}$, donde en el día 14 esta banda va tomando una forma más definida y su intensidad es un poco mayor comparada al día 11.

Posteriormente, se determinó las áreas relativas totales de las bandas FTIR de interés (fosfatos) tal como se muestra en el gráfico de la figura $3 a$, donde al tener la integral acumulada en cada uno de los puntos de cada espectro FTIR se calculó el área para cada banda tomando como Punto 1 (P1) el inicio de la banda y el Punto 2 (P2) su terminación, dicha área es igual a la resta (P2-P1) dividido el área total de todo el espectro, obteniendo un área total relativa de la banda de interés. Luego, al tener el área de cada banda de fosfato para cada espectro se suman cada una de estas áreas logrando así un total de área relativa acumulada. 
De este modo, el gráfico de la figura $3 b$, demuestra que hay una tendencia creciente en la pendiente de la curva, lo que indica que el biovidrio es bioactivo, es decir, por la biomineralización se empezó a depositar fosfato de calcio haciendo que estas moléculas se exciten e induzcan a la formación de una capa de apatita en la superficie del biovidrio.

Por otra parte, el gráfico de la figura 3c, describe el modelo cinético que mejor se ajustó al perfil de incremento de las áreas totales relativas de la figura $3 \mathrm{~b}$, donde la capacidad de biomineralización de los iones $\mathrm{PO}_{4}{ }^{3-}$ fue calculado mediante la ecuación de pseudo- primer-orden de Lagergren, representado como [16]:

$\ln \left(q_{e}-q_{t}\right)=\ln q_{e}-k_{1} t$

Donde en la Ec.(1), " $k_{1}$ " corresponde a la constante de equilibrio lineal de pseudo- primer-orden $\left(k_{1}=-\right.$ $0,2204)$, " $t$ " hace referencia al tiempo en los días de inmersión, " $q_{t}$ " es el equivalente a las áreas y " $q_{e}$ "

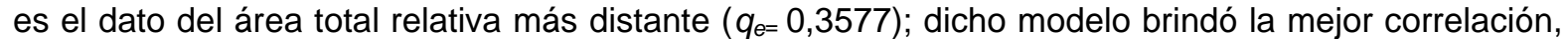
donde el coeficiente de determinación es de 0,9971 $\left(R^{2}>0,99\right)$. En efecto, el gráfico de la figura 3c, indica que la velocidad de biomineralización es lineal a la cantidad de fosfato presentes en la solución de SBF, por lo que los grupos activos del biovidrio proporcionaron suficientes sitios de nucleación y se acortó el periodo de inducción, dando lugar al crecimiento o adherencia de los iones de fosfato y calcio en la superficie del biovidrio, incitando a su vez la formación de una capa de apatita (rica en iones de $\mathrm{Ca}^{2+}$ y $\mathrm{PO}_{4}^{3-}$ ), por tal razón, el biomaterial es bioactivo.

a)

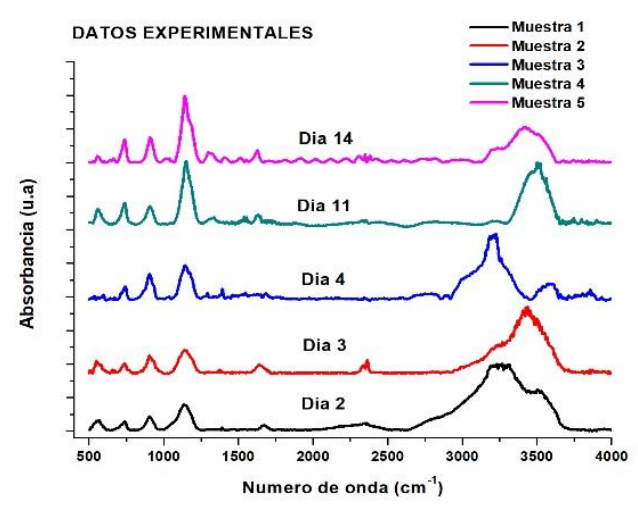

b)

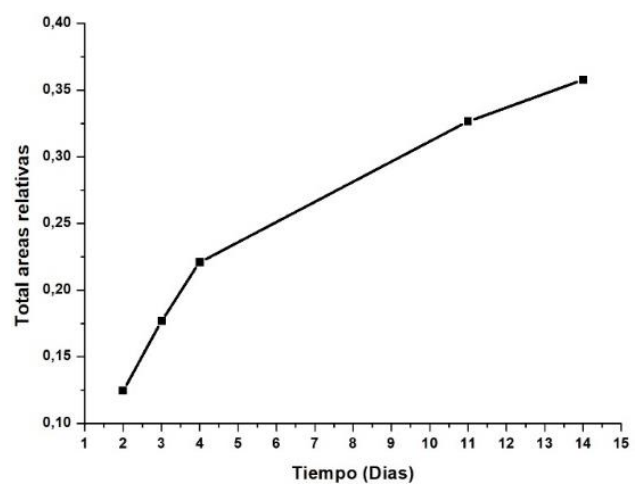

c)

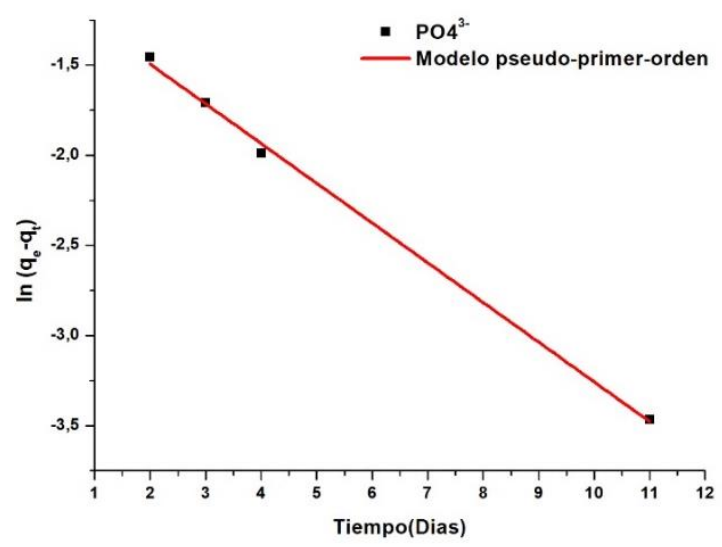

Figura 3. a) Evolución de los espectros infrarrojos de las muestras en los diferentes días de inmersión en SBF. Las bandas principales se encuentran en un rango alrededor de los 500 a $1400 \mathrm{~cm}^{-1}$, alusivas a bandas de fosfato; b) gráfico del incremento de las áreas totales relativas de las bandas de fosfato; c) cinética de adsorción de $\mathrm{PO}_{4}{ }^{3-}$ en el biovidrio durante el proceso de biomineralización representado por medio de un modelo cinético de pseudo- primer-orden. 


\section{CONCLUSIONES}

En este trabajo se sintetizó un biovidrio de fosfato de calcio por el método no convencional denominado pirólisis de aerosol en llama, posibilitando la síntesis de precursores en un tiempo rápido y obteniendo polvos micrométricos de biovidrio. La naturaleza amorfa del biovidrio fue confirmada por los resultados del DRX, encontrándose la presencia de la fase whitlockita. Por otro lado, el biovidrio mostró ser bioactivo presentando una cinética de biomineralización de pseudo- primer-orden.

\section{AGRADECIMIENTOS}

Al Instituto Tecnológico Metropolitano (I.T.M) por el soporte y apoyo para la realización de este estudio.

\section{REFERENCIAS BIBLIOGRÁFICAS}

[1] A.C. Colorado, C.A. Agudelo, M.E. Moncada A, Análisis de Biomateriales para uso en ingeniería de tejidos de piel, Rev. Ing. Biomed. 7 (2013) 11-23. https://doi.org/10.14508/rbme.2013.7.14.11-23.

[2] Maya Toribio Olea, Síntesis y caracterización de nanopartículas de fosfato de calcio para la regeneración de tejidos , Universidad Politécnica de Cataluña, 2014. http://hdl.handle.net/2099.1/23657.

[3] Kaur, O.P. Pandey, K. Singh, D. Homa, B. Scott, G. Pickrell, A review of bioactive glasses: Their structure, properties, fabrication and apatite formation, J. Biomed. Mater. Res. - Part A. 102 (2014) 254-274. https://doi.org/10.1002/jbm.a.34690.

[4,6,15] M.E. Navarro, Desarrollo y Caracterización de Materiales Biodegradables para Regeneración Ósea, Universidad Politécnica de Cataluña, 2005. http://hdl.handle.net/2117/93360.

[5] J.A. Perez Gonzalez, Síntesis de biovidrios por la técnica sol-gel con incorporación de metales y estudio de sus propiedades antibacteriales, Universidad de Chile, 2012. http://repositorio.uchile.cl/handle/2250/111122.

[7] S. Ataol, A. Tezcaner, O. Duygulu, D. Keskin, N.E. Machin, Synthesis and characterization of nanosized calcium phosphates by flame spray pyrolysis, and their effect on osteogenic differentiation of stem cells, J. Nanoparticle Res. 17 (2015). https://doi.org/10.1007/s11051-015-2901-0.

[8] T.S. Lyubenova, J.B. Carda, M. Ocaña, Synthesis by pyrolysis of aerosols and ceramic application of Cr-doped CaYAlO4 red-orange pigments, J. Eur. Ceram. Soc. 29 (2009) 2193-2198. https://doi.org/10.1016/j.jeurceramsoc.2009.01.020.

[9] E. López-Navarrete, A.R. González-Elipe, M. Ocaña, Non-conventional synthesis of Cr-doped SnO2 pigments, Ceram. Int. 29 (2003) 385-392. https://doi.org/10.1016/S0272-8842(02)00149-9.

[10] N. Betancur Granados, Síntesis de pigmentos cerámicos a partir de cromitas de estructura tipo espinela por el método no convencional de pirólisis de aerosol con llama con alimentación líquida, Universidad Nacional de Colombia, 2015. https://repositorio.unal.edu.co/handle/unal/53052.

[11] N. Betancur Granados, SYNTHESIS OF CALCIUM SILICATES BY FLAME SPRAY PYROLYSIS, Univer-sidad Nacional de Colombia, 2019. https://repositorio.unal.edu.co/handle/unal/75580.

[12] T. Kokubo, H. Takadama, How useful is SBF in predicting in vivo bone bioactivity?, Biomaterials. 27 (2006) 2907-2915. https://doi.org/10.1016/j.biomaterials.2006.01.017. 
[13] E. Ciro, N. Zapata, E. López, Cerámica y Vidrio, Boletín La Soc. Española Cerámica y Vidr. 54 (2015) 84-92. https://doi.org/10.1016/j.bsecv.2015.03.006.

[14] H.A. Elbatal, M.A. Azooz, E.M.A. Khalil, A.S. Monem, Y.M. Hamdy, Characterization of some bioglass - ceramics, 80 (2003) 599-609. https://doi.org/10.1016/S0254-0584(03)00082-8.

[16] M. Xia, P. Bao, A. Liu, L. Shen, R. Yu, Y. Liu, J. Li, Colloids and Surfaces B: Biointerfaces Application of the kinetic and isotherm models for better understanding of the mechanism of biomineralization process induced by Purpureocillium lilacinum Y3, 181 (2019) 207-214. https://doi.org/10.1016/j.colsurfb.2019.05.051. 